\title{
A Monte Carlo Radiation Model for Simulating Rarefied Multiphase Plume Flows
}

\author{
Jonathan M. Burt ${ }^{*}$ and Iain D. Boyd ${ }^{\dagger}$ \\ Department of Aerospace Engineering \\ University of Michigan, Ann Arbor, MI 48109
}

\begin{abstract}
A Monte Carlo ray trace radiation model is presented for the determination of radiative properties of $\mathrm{Al}_{2} \mathrm{O}_{3}$ particles in the high altitude plume of a solid propellant rocket. A polydisperse distribution of non-gray particles is modeled as an emitting, absorbing and scattering medium of arbitrary optical thickness. Strong two-way coupling is allowed between radiation and flowfield calculations, where the gas is simulated using the direct simulation Monte Carlo method and particle phase properties are determined using a similar Lagrangian approach. Effects of anisotropic scattering and nozzle searchlight emission are considered, and a procedure is described for the calculation of spectral radiance. The model is applied to the simulation and radiation analysis of the freely expanding plume from a subscale solid rocket motor, and various flowfield properties are presented and discussed.
\end{abstract}

\section{Introduction}

$\mathrm{T}$ HE analysis and prediction of radiation signatures from solid propellant rocket plumes has been the subject of extensive study over the past several decades. ${ }^{1}$ Much work has focused on properties of micron-scale $\mathrm{Al}_{2} \mathrm{O}_{3}$ particles which account for up to $30 \%$ of the mass flow through the nozzle, and which tend to dominate IR radiative properties within the plume. Characteristics of the particle phase are extremely complex, and are generally either poorly understood or difficult to model within a numerical simulation. Of particular interest here are the particle phase properties in plume flows at very high altitudes, where interactions between the particles and gas are both important and not well suited to traditional simulation techniques.

In a typical high altitude exhaust flow from a solid rocket motor (SRM), liquid aluminum droplets are formed along the propellant grain surface, and undergo a complicated process of combustion, vaporization, agglomeration, breakup, and crystallization as they are forced by the expanding gas through the combustion chamber and nozzle. ${ }^{2}$ At the nozzle exit, the particle phase consists of a polydisperse distribution of spherical $\mathrm{Al}_{2} \mathrm{O}_{3}$ particles, which range in diameter from about 0.1 to $10 \mu \mathrm{m}$ depending on the size of the nozzle. Larger particles exist mainly in liquid form within the nozzle and tend to develop greater temperature and velocity lags relative to the gas. The smallest particles are rapidly accelerated and cooled by the surrounding gas, and will have fully solidified into some combination of metastable gamma phase and stable alpha phase polycrystalline structures as they pass through the nozzle exit plane.

Within the plume nearfield region, the crystallization process becomes increasingly important for particles of intermediate size, for which radiative and convective cooling are balanced by a heat release during the phase transition process. The interaction between the gas and particles in this region is both significant and complex, as the gas develops a high degree of thermal nonequilibrium during the rapid expansion downstream of the nozzle exit. Due to large particle phase velocity and temperature lags, the interphase transfer of momentum and energy may greatly affect particle properties, while the considerable particle mass fraction allows bulk gas properties to be influenced by gas-particle collisions. Further downstream, interphase momentum and energy exchange become negligible as the gas continues to expand. The largest particles may begin to solidify far downstream of the nozzle, where radiative heat transfer from and between particles tends to dominate the particle phase energy balance. Depending on the SRM size and grain composition, the farfield plume regions will likely have some intermediate optical thickness, so that long-range radiative energy exchange within the particle phase may significantly influence particle temperatures.

\footnotetext{
* Graduate student, AIAA student member.

${ }^{\dagger}$ Professor, AIAA associate fellow.
} 
For accurate prediction of the radiation signature from a high altitude SRM plume flow, several of these processes must be considered, and the nonequilibrium nature of the gas requires a simulation approach which can overcome limitations of traditional computational fluid dynamics (CFD) techniques. Various complex radiative properties of multiphase particles should be accounted for, including a temperature, size, and wavelength dependence in spectral emissivities. Emission, absorption, and scattering may all be important, and strong two-way coupling can exist between the radiation field and particle temperatures. This last property may produce large errors in the traditional post-processing approach to plume radiation analysis. With these goals in mind, a new procedure is proposed for the simulation and radiation analysis of high altitude SRM plume flows.

Several steps in this procedure have been extensively documented in recent papers, so are only discussed here in general terms. The gas is simulated using the Direct Simulation Monte Carlo (DSMC) method of Bird, ${ }^{3}$ and the solid phase is modeled using a similar Lagrangian approach in which representative particles are tracked through the computational grid. Momentum and energy transfer to a particle from the surrounding gas is modeled through a Green's function technique of Gallis et al., ${ }^{4}$ where the total force and heat transfer rate is calculated during each time step by summing contributions from all DSMC gas molecules assigned to the same grid cell. The reciprocal momentum and energy transfer from the particle phase to the gas is computed using a probabilistic approach, in which individual interphase collisions are modeled as involving either specular reflection or diffuse reflection with full thermal accommodation., 5 Several additional capabilities and physical models have been developed and implemented in the DSMC code MONACO. ${ }^{7}$ Mixed discrete and continuous particle size distributions may be used, and consideration is made for the effects of particle rotation, nonspherical particles, the breakup of liquid droplets and nonequilibrium crystallization. In addition, a series of interphase coupling parameters ${ }^{8}$ are utilized to increase computational efficiency, through a process involving the automatic determination of flowfield regions where calculations for momentum and energy transfer in either direction may be avoided with negligible impact on bulk flow properties.

In the following sections, a particle radiation model is presented for use with the above procedures in the simulation of a high altitude SRM plume flow. First, a detailed description of the method is given, including discussion of procedures related to emission, absorption, scattering and two-way coupling between radiation and flowfield characteristics. In addition, techniques are outlined for consideration of nozzle searchlight emission and the calculation of spectral radiance. The radiation model is then applied to the plume simulation for a representative subscale SRM exhausting into a vacuum. Simulation results are discussed, and sensitivities of radiation characteristics to various input parameters are evaluated. In particular, we consider the effects of nozzle searchlight emission and anisotropic scattering on spectral radiance and the net radiative energy flux.

\section{Radiation Modeling Procedure}

The proposed particle radiation model uses a Monte Carlo Ray Trace (MCRT) approach, where a Lagrangian representation is used to track large groups of photons through the computational grid., ${ }^{9,10}$ As a first step, the portion of the spectrum of interest for radiative heat transfer - wavelengths of roughly 0.5 to $5 \mu \mathrm{m}$ - is divided into a series of wave number bins. Given $N_{\eta}$ different bins, each of width $\Delta \eta_{i}$ and centered at a wave number $\eta_{i}$, a large number $\mathrm{N}_{\mathrm{b}}$ of representative "energy bundles" are generated once every several time steps at randomly selected source particles throughout the grid. Every bundle represents some quantity of radiative power, and an equal number of bundles is assigned to each of the $N_{\eta}$ bins. A newly generated bundle is given a direction of propagation according to a randomly generated unit vector $\mathbf{u}$. The bundle is also given some initial power $\mathrm{P}_{\mathrm{b}}$, based on the assigned wave number bin and the properties of the source particle.

Following a correlation of Reed and Calia ${ }^{1}$ based on Mie theory calculations of Plass, ${ }^{11}$ the band-averaged spectral emissivity of the source particle at bin i may be approximated as

$$
\varepsilon_{\mathrm{i}}=4 \mathrm{k}\left(\mathrm{T}_{\mathrm{p}}, \eta_{\mathrm{i}}\right) \mathrm{R}_{\mathrm{p}} \eta_{\mathrm{i}}
$$

where $T_{p}$ and $R_{p}$ are the temperature and radius of the particle respectively, and $k$ is a value for the absorption index of $\mathrm{Al}_{2} \mathrm{O}_{3}$ at temperature $\mathrm{T}_{\mathrm{p}}$ and wave number $\eta_{\mathrm{i}}$. Due to both a lack of experimental data and an extreme sensitivity of $\mathrm{k}$ for solid particles on lattice defects and impurities, ${ }^{1}$ we neglect any dependence of $\mathrm{k}$ on the particle phase composition. By applying Eq. (1) to Planck's blackbody function, we can compute the source particle emissive power $\mathrm{P}_{\mathrm{p}, \mathrm{i}}$ within the wave number range to which the bundle is assigned:

$$
\mathrm{P}_{\mathrm{p}, \mathrm{i}}=32 \pi^{2} \mathrm{c}_{0}^{2} \mathrm{~h} \mathrm{R}_{\mathrm{p}}^{3} \Omega_{\mathrm{i}}\left(\mathrm{T}_{\mathrm{p}}\right)
$$


where

$$
\Omega_{\mathrm{i}}(\mathrm{T})=\int_{\Delta \eta_{\mathrm{i}}} \frac{\mathrm{k}\left(\mathrm{T}, \eta_{\mathrm{i}}\right) \eta^{4} \mathrm{~d} \eta}{\exp \left(\mathrm{hc}_{0} \eta / \mathrm{k}_{\mathrm{B}} \mathrm{T}\right)-1}
$$

The symbol $\mathrm{c}_{\mathrm{o}}$ here is the speed of light through a vacuum, $\mathrm{h}$ is Planck's constant, and $\mathrm{k}_{\mathrm{B}}$ is Boltzmann's constant. The initial power of the bundle is then determined as

$$
\mathrm{P}_{\mathrm{b}}=\frac{\mathrm{N}_{\eta} \mathrm{N}_{\mathrm{p}}}{\mathrm{N}_{\mathrm{b}}} \mathrm{W}_{\mathrm{p}} \mathrm{P}_{\mathrm{p}, \mathrm{i}}
$$

where $\mathrm{N}_{\mathrm{p}}$ is the total number of representative particles in the grid and $\mathrm{W}_{\mathrm{p}}$ is the numerical weight of the source particle. Values of $\Omega_{\mathrm{i}}(\mathrm{T})$ are calculated at simulation startup for each wave number bin, at temperatures $\mathrm{T}$ for which experimentally determined $k\left(T, \eta_{i}\right)$ values are available. The evaluation of $\Omega_{i}\left(T_{p}\right)$ in Eq. (2) is then performed through linear interpolation to the particle temperature.

Once created, each energy bundle is tracked through the grid during the current time step until it exits through an inflow, outflow, or absorbing wall boundary. As an energy bundle passes through a cell in which particles are located (or have been located during previous time steps) a fraction of the assigned power will be absorbed, and there is some probability that the bundle will be scattered. Absorption and scattering properties in the cell for a given wave number bin are determined by the band-averaged spectral absorptance $\alpha_{i}$ and scattering coefficient $\sigma_{\mathrm{i}}$. Consider a simulation involving $\mathrm{N}_{\text {spec }}$ different particle species $\mathrm{j}$, where species are designated according to the particle radius $R_{j}$. In the cell of interest, each species has an average temperature $T_{j}$ and a number density $n_{j}$, where averaging is performed over a large number of time steps for both $T_{j}$ and $n_{j}$. By applying Eq. (1) and Kirchoff's law to the definition of spectral absorptance, we can compute $\alpha_{i}$ as

$$
\alpha_{i}=4 \pi \eta_{i} \sum_{j=1}^{N_{\text {spec }}} k\left(T_{j}, \eta_{i}\right) R_{j}^{3} n_{j} .
$$

The value of $k\left(T_{j}, \eta_{i}\right)$ for each species is found by interpolating tabulated $k$ values to the temperature $T_{j}$. The corresponding scattering coefficient $\sigma_{\mathrm{i}}$ is also given as a summation over all particle species:

$$
\sigma_{\mathrm{i}}=\pi \sum_{\mathrm{j}=1}^{\mathrm{N}_{\mathrm{spec}}} \Theta_{\mathrm{i}, \mathrm{j}} \mathrm{R}_{\mathrm{j}}^{2} n_{\mathrm{j}}
$$

The symbol $\Theta_{\mathrm{i}, \mathrm{j}}$ in Eq. (6) is the scattering efficiency factor for wave number bin i and particle species $\mathrm{j}$. Values of $\Theta_{i, j}$ are calculated at simulation startup, using a first-order Mie theory approximation of Siegel and Howell. ${ }^{12}$ Assuming that $n_{\mathrm{i}}>>\mathrm{k}$, where $\mathrm{n}_{\mathrm{i}}$ is the real part of the index of refraction for the particle material at wave number $\eta_{\mathrm{i}}$, $\Theta_{i, j}$ may be given as a function of the nondimensional parameter $x_{i, j}=2 \pi \eta_{i} R_{j}$.

$$
\Theta_{i, j}=\frac{8}{3} x_{i, j}^{4}\left[\frac{n_{i}^{2}-1}{n_{i}^{2}+2}\left(1+\frac{3}{5}\left(\frac{n_{i}^{2}-2}{n_{i}^{2}+2}\right) x_{i, j}^{2}\right)\right]^{2}
$$

While Eq. (7) can be assumed accurate for $\mathrm{Al}_{2} \mathrm{O}_{3}$ when $\mathrm{x}_{\mathrm{i}, \mathrm{j}}<<1$, it greatly over predicts $\Theta_{\mathrm{i}, \mathrm{j}}$ for larger values of $\mathrm{x}_{\mathrm{i}, \mathrm{j}}$. To allow for use with a wider range of $x_{i, j}$ values, we impose a limiting condition $\Theta_{i, j} \leq 2$. This gives relatively good agreement with Mie theory calculations of Plass, ${ }^{11}$ and avoids the detailed calculations required to find an exact Mie theory solution. Note that we neglect here any dependence of $n_{i}$ on the particle temperature, following observations that $n_{i}$ values for $\mathrm{Al}_{2} \mathrm{O}_{3}$ are nearly constant over a wide range of temperatures. ${ }^{11}$ While experiments have shown some increase in $n_{\mathrm{i}}$ with particle size, ${ }^{13}$ we neglect this dependence as well due to a lack of available experimental data. 
When an energy bundle enters a given cell, the total distance $D_{e}$ to exit the cell along the initial trajectory is determined along with $\alpha_{i}$ and $\sigma_{i}$ values corresponding to the assigned wave number bin. The distance $D_{s}$ to a scattering event is then determined by evaluating the probability $P_{\mathrm{ns}}$ that the bundle will not have been scattered after it has traveled a distance $\mathrm{D}_{\mathrm{s}}$, where

$$
\frac{d P_{\mathrm{ns}}}{d \mathrm{D}_{\mathrm{s}}}=-\sigma_{\mathrm{i}} P_{\mathrm{ns}}
$$

Solving for $\mathrm{D}_{\mathrm{s}}$ and setting $P_{\mathrm{ns}}$ equal to a random number $\Re \in[0,1]$, we find

$$
\mathrm{D}_{\mathrm{s}}=-\frac{1}{\sigma_{\mathrm{i}}} \ln \Re .
$$

If $D_{s}>D_{e}$ then the particle will exit the cell along its initial trajectory. Otherwise the bundle will be scattered. If scattered, the bundle is moved a distance $\mathrm{D}_{\mathrm{s}}$ along the trajectory, after which its direction is reassigned and the procedure is repeated.

The anisotropic nature of the scattering process is approximated through the use of the Henyey Greenstein scattering phase function ${ }^{14}$

$$
\Phi(\theta)=\frac{1-g^{2}}{4 \pi\left(1+g^{2}-2 g \cos \theta\right)^{3 / 2}} .
$$

The free parameter g in Eq. (10) is the average cosine of the scattering angle $\theta$. We can recover the corresponding distribution function $f(\theta)=2 \pi \Phi(\theta) \sin \theta$ if we set

$$
\mathfrak{R}=2 \pi \int_{0}^{\theta} \Phi\left(\theta^{\prime}\right) \sin \theta^{\prime} d \theta^{\prime}
$$

for a random number $\Re$ in between 0 and 1 . Following Eqs. (10) and (11), we determine $\theta$ through the formula

$$
\cos \theta=\frac{1}{2 g}\left[g^{2}+1-\left(\frac{1-g^{2}}{2 g \Re-g-1}\right)^{2}\right] \text {. }
$$

The unit vector $\mathbf{u}^{*}$ in the final direction of propagation is then calculated as

$$
\mathbf{u}^{*}=\mathbf{u} \cos \theta+\mathbf{t}_{1} \sin \theta \cos \phi+\mathbf{t}_{2} \sin \theta \sin \phi
$$

where the azimuthal angle $\phi$ is assigned a random value in $[0,2 \pi]$, $\mathbf{u}$ is the initial direction, and the unit vectors $\mathbf{t}_{1}$ and $\mathbf{t}_{2}$ are given by

$$
\mathbf{t}_{1}=\frac{\mathbf{u} \times \hat{\mathbf{i}}}{|\mathbf{u} \times \hat{\mathbf{i}}|} \text { and } \mathbf{t}_{2}=\mathbf{t}_{1} \times \mathbf{u} .
$$

For convenience, $\hat{\mathbf{i}}$ is defined here as the unit vector along the x-coordinate axis.

The procedure involving the evaluation of Eqs. (9), (12) and (13) is repeated until the bundle exits the cell. Given a total distance $D_{t}$ which the bundle has traveled through the cell, the assigned power $P_{b}$ is then reduced by a fraction 1-exp $\left(-\alpha_{i} D_{t}\right)$ to account for the effect of particle phase absorption on the transmitted radiation intensity. ${ }^{9}$

As the bundle passes through the cell, the power $\Delta \mathrm{Q}_{\text {abs }}$ absorbed by an individual particle of radius $\mathrm{R}_{\mathrm{p}}$ may be given as 


$$
\Delta \mathrm{Q}_{\mathrm{abs}}=\frac{\pi \mathrm{R}_{\mathrm{p}}^{2} \varepsilon_{\mathrm{i}}}{\alpha_{\mathrm{i}} \mathrm{V}_{\text {cell }}} \mathrm{P}_{\mathrm{b}}\left[1-\exp \left(-\alpha_{\mathrm{i}} \mathrm{D}_{\mathrm{t}}\right)\right]
$$

where $V_{\text {cell }}$ is the cell volume and $P_{b}$ is the initial power assigned to the bundle on entering the cell. It follows that the contribution of the bundle to the direction-averaged radiative energy flux $\Delta q_{\mathrm{i}}$ for the corresponding wave number bin is

$$
\Delta q_{\mathrm{i}}=\frac{\mathrm{P}_{\mathrm{b}}}{\alpha_{\mathrm{i}} \mathrm{V}_{\text {cell }}}\left[1-\exp \left(-\alpha_{\mathrm{i}} \mathrm{D}_{\mathrm{t}}\right)\right]
$$

In an optically thin cell where $\alpha_{\mathrm{i}} \mathrm{D}_{\mathrm{t}}<<1$ the evaluation of Eq. (15) may result in a large subtractive cancellation error. To correct for this error, when $\alpha_{\mathrm{i}} \mathrm{D}_{\mathrm{t}}<10^{-5}$ we calculate $\Delta q_{\mathrm{i}}$ using a linearized form of Eq. (15)

$$
\Delta q_{\mathrm{i}}=\frac{\mathrm{P}_{\mathrm{b}} \mathrm{D}_{\mathrm{t}}}{\mathrm{V}_{\text {cell }}}
$$

based on a Taylor expansion of the exponential term. Note that Eq. (16) gives an exact solution for $\Delta q_{i}$ in regions outside the particle domain where $\alpha_{\mathrm{i}}$ is zero. During each time step for which energy bundles are tracked through the grid, energy flux values in every cell are determined by summing contributions $\Delta q_{\mathrm{i}}$ from all bundles which pass through the cell. The resulting values are then averaged over a large number of time steps to reduce statistical scatter. Once the flowfield has reached steady state conditions, averaging may be performed over all subsequent time steps during which radiation calculations are made. Otherwise a subrelaxation technique of Sun and Boyd ${ }^{15}$ is used for the time-averaging procedure, so that increased weighting may be applied to more recent time steps.

As discussed above, strong two-way coupling may exist between flowfield characteristics and plume radiation. Radiative heat transfer can significantly affect particle temperatures, and may indirectly influence other properties such as the particle phase composition, material density, and the rates of momentum and energy transfer between the particles and gas. To account for the effect of radiative emission and absorption on particle temperatures, the temperature $T_{p}$ of every representative particle is modified during each time step by

$$
\Delta \mathrm{T}_{\mathrm{p}}=\frac{\Delta \mathrm{t}}{\mathrm{m}_{\mathrm{p}} \mathrm{c}_{\mathrm{p}}} \mathrm{Q}_{\mathrm{rad}}
$$

where $\Delta t$ is the time step interval, $m_{p}$ is the particle mass, $c_{p}$ is the specific heat of the particle material, and $Q_{\text {rad }}$ is the net rate of radiative heat transfer to the particle. Note that the particle temperature is assumed spatially uniform, based on a low Biot number approximation which follows from the small particle size and relatively high thermal conductivity of $\mathrm{Al}_{2} \mathrm{O}_{3}$. The radiative heat transfer rate is calculated as

$$
\mathrm{Q}_{\mathrm{rad}}=4 \pi \mathrm{R}_{\mathrm{p}}^{3} \sum_{\mathrm{i}=1}^{\mathrm{N}_{\mathrm{\eta}}}\left[\eta_{\mathrm{i}} \mathrm{k}\left(\mathrm{T}_{\mathrm{p}}, \eta_{\mathrm{i}}\right) q_{\mathrm{i}}-8 \pi \mathrm{c}_{0}^{2} \mathrm{~h} \Omega_{\mathrm{i}}\left(\mathrm{T}_{\mathrm{p}}\right)\right]
$$

following Kirchoff's law and Eqs. (1) and (2). The above symbol $q_{\mathrm{i}}$ is the time-averaged and direction-averaged energy flux within a wave number bin $\mathrm{i}$, for the cell in which the particle is located.

Radiative emission from within the nozzle has been found to significantly increase radiance values near the exit plane. ${ }^{16}$ This emission is generated primarily by the inner nozzle walls, and is generally termed "searchlight emission" under past assumptions that its dominant source is upstream of the throat. Depending on the optical thickness of the exhaust flow, emission from particles within the nozzle may also contribute significantly. As searchlight emission is expected to influence the temperature and phase composition of particles in the plume, a coupled approach to radiation and flowfield simulation should include consideration of this effect. We account for searchlight emission through the generation of additional energy bundles along inflow boundaries at the nozzle exit.

Each inflow boundary on the exit plane is represented as a blackbody wall at some characteristic temperature $T_{w}$. Along every cell face located on an inflow boundary, $\mathrm{N}_{\mathrm{f}}$ new bundles are generated during each time step for which

American Institute of Aeronautics and Astronautics Approved for public release; distribution unlimited. 
radiation calculations are performed. Each bundle is randomly assigned to a wave number bin i, and is given an initial direction $\mathbf{u}$ such that

$$
\mathbf{u} \cdot \mathbf{n}_{\mathrm{f}}=\cos \theta=\mathfrak{R}^{1 / 2}
$$

where $\mathbf{n}_{\mathrm{f}}$ is the inward normal unit vector at the cell face, $\theta$ is the zenithal angle of $\mathbf{u}$ relative to the face, and $\Re$ is a random number in $[0,1]$. The initial power $P_{b}$ is then determined from Eq. (19) as the product of the face area $A_{f}$, a weighting factor $\mathrm{N}_{\mathrm{\eta}} / \mathrm{N}_{\mathrm{f}}$, and the integral of Planck's function over the corresponding wave number bin.

$$
\mathrm{P}_{\mathrm{b}}=2 \pi \mathrm{c}_{0}^{2} h \mathrm{~A}_{\mathrm{f}} \frac{\mathrm{N}_{\eta}}{\mathrm{N}_{\mathrm{f}}} \int_{\Delta \eta_{\mathrm{i}}} \frac{\eta^{3} \mathrm{~d} \eta}{\exp \left(\mathrm{hc}_{0} \eta / \mathrm{k}_{\mathrm{B}} \mathrm{T}_{\mathrm{w}}\right)-1}
$$

For calculations of plume radiance, one or more simulated radiometers are positioned somewhere outside the grid domain. Consider a sensor with surface area $A_{s}$, outward unit normal $\mathbf{n}_{s}$, and angular resolution defined by the zenithal angle $\omega$. When each energy bundle exits the grid, we determine whether it will intersect the sensor surface along a trajectory given by the unit vector $\mathbf{u}$ for the bundle direction. If an intersection occurs and the condition

$$
-\mathbf{u} \cdot \mathbf{n}_{\mathrm{s}} \geq \cos \omega
$$

is met, then the power $\mathrm{P}_{\mathrm{b}}$ assigned to the bundle is added to the total absorbed power $\Sigma \mathrm{P}_{\mathrm{i}}$ for the corresponding wave number bin during the current time step. An instantaneous value of the band-averaged spectral radiance $\mathrm{I}_{\mathrm{i}}$ may then be calculated as the ratio of $\Sigma \mathrm{P}_{\mathrm{i}}$ to the product of the sensor area, solid angle for absorbed radiation, and wavelength range of the bin. We find

$$
\mathrm{I}_{\mathrm{i}}=\frac{\Sigma \mathrm{P}_{\mathrm{i}}}{2 \pi(1-\cos \omega) \mathrm{A}_{\mathrm{s}} \Delta \lambda_{\mathrm{i}}}
$$

where

$$
\Delta \lambda_{\mathrm{i}}=\frac{\Delta \eta_{\mathrm{i}}}{\eta_{\mathrm{i}}^{2}-\frac{1}{4}\left(\Delta \eta_{\mathrm{i}}\right)^{2}}
$$

Values of $I_{i}$ for each bin are averaged over a large number of time steps to reduce scatter, where sampling is begun only after the flowfield has reached steady state conditions. Note that the rate of statistical convergence in the resulting radiance values will be roughly equal for every bin. While a much faster convergence rate is possible if we apply a reverse Monte Carlo method involving the generation of additional energy bundles at the sensor, ${ }^{14}$ the procedure proposed here allows for relatively fast convergence without adding much to the complexity of the radiation model implementation.

\section{Plume Flow Test Case}

Following implementation in the DSMC code MONACO, ${ }^{7}$ the procedures described above are applied to an axisymmetric test case for the plume flow from a subscale SRM expelling into a vacuum. Inflow and boundary conditions are identical to those described in a previous paper, ${ }^{8}$ as are all physical models used in the flowfield simulation. We use a rectangular grid geometry extending from the nozzle exit plane $100 \mathrm{~m}$ downstream and $40 \mathrm{~m}$ radially outward. The nozzle exit diameter is $7.85 \mathrm{~cm}$, and exit plane data for both the particles and gas are taken from Anfimov et al. ${ }^{17}$ based on simulated nozzle flow characteristics for a Star-27 motor with $30 \%$ particle mass loading. The gas is a mixture of $\mathrm{N}_{2}, \mathrm{H}_{2}$ and CO, and the variable hard sphere (VHS) collision model ${ }^{3}$ is used for collisions within the gas phase. At the exit plane, the gas is assigned a bulk speed of $3113 \mathrm{~m} / \mathrm{s}$, a temperature of $1433 \mathrm{~K}$ and a density of $0.011 \mathrm{~kg} / \mathrm{m}^{3}$, with mole fractions of 0.38 for $\mathrm{H}_{2}$ and 0.31 for both $\mathrm{N}_{2}$ and $\mathrm{CO}$.

The particle phase has a discrete size distribution, with seven different species ranging in diameter from 0.3 to 6 $\mu \mathrm{m}$. Due to a lack of available flowfield information at the nozzle exit, particle properties given by Anfimov et al. ${ }^{17}$ 
are assumed uniform over the exit plane. A rough estimate of the initial solid mass fraction for each particle size is found by taking the difference between the nucleation temperature $(1930 \mathrm{~K})$ for homogeneous crystallization of $\mathrm{Al}_{2} \mathrm{O}_{3}$ and the assigned particle temperature, then multiplying this by the ratio of the specific heat to the latent heat of fusion for liquid $\mathrm{Al}_{2} \mathrm{O}_{3}$. Particle properties at the nozzle exit are given in Table 1.

The thermal accommodation coefficient on the particle surface is set to 0.9 , so that $90 \%$ of interphase collisions involve diffuse reflection with full accommodation to the particle temperature, while the remaining $10 \%$ involve specular reflection. Interphase momentum and energy transfer is computed using the two-way coupling approach described above, and the crystallization of liquid $\mathrm{Al}_{2} \mathrm{O}_{3}$ droplets is considered using a model for nonequilibrium phase change. ${ }^{8}$ The phase change model accounts for a temperature dependence in the crystallization rate and the associated heat release, while neglecting the gamma-to-alpha transition for solid $\mathrm{Al}_{2} \mathrm{O}_{3}$ and the density variation between different phases.

Values of the particle absorption index $\mathrm{k}$ are taken from experimental data of Konopka, Reed and Calia. ${ }^{18}$ Based on this data, we use 10 wave number bins corresponding to the mid-IR wavelength range from 1.3 to $4.5 \mu \mathrm{m}$. Of the two SRM exhaust flows from which particles were collected and investigated by these authors, the second (rocket 2) was found to give $\mathrm{k}$ values more in line with other experimental data and correlations in the literature. ${ }^{19}$ Values calculated from SEM measurements for the second flow are therefore used here. The real part $n_{i}$ of the particle index of refraction is computed as a function of $\eta_{i}$ through a correlation given by Duval et al., ${ }^{19}$

$$
\mathrm{n}_{\mathrm{i}}=\left(0.9904+2.02 \times 10^{-5} \mathrm{~T}_{\mathrm{p}}\right)\left[1+\frac{1.024}{1-0.00376 \eta_{\mathrm{i}}^{2}}+\frac{1.058}{1-0.01225 \eta_{\mathrm{i}}^{2}}+\frac{5.281}{1-321.4 \eta_{\mathrm{i}}^{2}}\right]^{1 / 2}
$$

where $\eta_{i}$ is in units of $\mu \mathrm{m}^{-1}$ and the weak temperature dependence is neglected by assuming a particle temperature $T_{p}$ of $2000 \mathrm{~K}$.

Following Reed et al., ${ }^{16}$ we set the average cosine of the scattering angle to $g=0.5$ and use an effective temperature of $\mathrm{T}_{\mathrm{w}}=1300 \mathrm{~K}$ for searchlight emission at the nozzle exit. The unstructured grid consists of approximately 40,000 triangular cells scaled roughly according to the local mean free path. So that calculations may be performed in a reasonable amount of time on available computing resources, cells are about 10 to 50 times larger than the mean free path in a small region just beyond the nozzle exit. While this is a violation of the standard rule of thumb for cell size in DSMC, we can tolerate the associated errors with an understanding that the simulation performed here is intended less to represent one particular flow with maximum accuracy than to show the general radiation characteristics of a typical small-scale SRM plume flow at high altitude.

About two million DSMC gas molecules and 200,000 representative solid particles are tracked through the grid during a typical time step at steady state. Sampling is performed once every five time steps, when 1600 energy bundles are generated and moved through the grid. To reach the desired level of statistical scatter in sampled radiation and bulk flow properties, the simulation is run for 100 hours on four processors in a $1.4 \mathrm{GHz}$ AMD Athlon cluster. Calculations are divided roughly evenly across all processors through domain decomposition, and buffer arrays are used to allow energy bundles to rapidly move across multiple task domains while restricting information exchange between neighboring tasks. Selected simulation results are shown in Figs. 1 through 8.

\section{Simulation Results}

Mass density contours for both particles and gas are shown in Fig. 1. Note first that particles are only found in roughly half of the simulation domain, as the maximum divergence angle for the particle phase is restricted by particle mass. All particles are given initial trajectories between 0 and 15 degrees off the centerline at the nozzle exit plane, where the trajectory angle for each particle scales linearly with distance from the axis. In the plume nearfield region just beyond the nozzle exit, particles are forced outward from the centerline by the expanding gas. The radial acceleration of an individual particle in this region will vary as the inverse of the particle diameter, so that in a given radial plane smaller particles will be found over a range which extends further from the axis. As shown in the top half of the figure, this results in a gradual decrease in particle mass density with distance from the axis due to the presence of a range of particle sizes.

As the dimensions of the simulation domain are several orders of magnitude greater than the nozzle exit radius, particle characteristics observed in Fig. 1 reflect only trends in the farfield region, where momentum and energy transfer between the particles and gas can be assumed negligible. The particles move along nearly straight trajectories far from the nozzle, so the contour lines shown projecting from the nozzle exit are straight if we neglect effects of statistical scatter and interpolation between cell centers. The continuous reduction in particle mass density 
with downstream distance is due to the divergence of particle trajectories, and the restricted maximum divergence angle is shown in the figure to result in centerline values of the particle phase mass fraction (the ratio of particle mass density to total mass density for both particles and gas) which increase with distance from the nozzle. At the point on the centerline $100 \mathrm{~m}$ downstream of the nozzle exit, the particle mass fraction is about 2.2 times the initial value of 0.3 .

Figure 2 shows contours of average temperature for particles of diameter $0.4 \mu \mathrm{m}$ and $4 \mu \mathrm{m}$. The temperature of $0.4 \mu \mathrm{m}$ particles is generally observed to decrease with downstream distance but increase with distance from the axis. The first trend may be attributed to the dominance of radiative heat loss in the particle energy balance through nearly the entire simulation domain, while the second trend is primarily a result of the radial variation in gas density within the plume nearfield region. As gas streamlines diverge in this region just downstream of the nozzle exit, the density of the gas will decrease more rapidly along streamlines further from the centerline. A lower gas density corresponds to a reduction in the rate of convective heat transfer from particles to the surrounding gas, so we can expect a smaller temperature drop in the plume nearfield for particles which pass through the nozzle exit plane further from the centerline. This is likely the dominant mechanism for the increase in particle temperature with distance from the axis.

Another possible contributor to this trend is the fact that particles further from the axis may absorb more radiative energy from nozzle searchlight emission. The plume optical thickness scales with particle phase mass density, and this density is shown in Fig. 1 to decrease with distance from the axis, so a corresponding increase in the magnitude of long range radiative intensity from nozzle emission should result in some increase in absorption among particles far from the centerline. However, this trend is found to have only a very small effect on particle temperatures, and is countered by an increase in short range radiative heat transfer between particles near the axis. We expect short range radiative transfer to be the dominant mechanism by which radiation causes a radial variation in particle temperatures, so that the net effect of coupling the radiation model to the flowfield simulation should be a slight reduction in the temperature increase for $0.4 \mu \mathrm{m}$ particles with distance from the axis. Note that a significant temperature drop is observed for these particles at points very close to the axis. This is likely the result of an unphysical decrease in convective heat transfer between the particles and gas, which follows from a reduction in the average number of DSMC gas molecules in cells along the centerline just downstream of the nozzle exit.

Similar trends as described above are found within $50 \mathrm{~m}$ of the nozzle exit for $4 \mu \mathrm{m}$ diameter particles, as shown in the lower half of Fig. 2. Average temperatures for these particles in the first $50 \mathrm{~m}$ downstream of the nozzle are several hundred degrees higher than for the $0.4 \mu \mathrm{m}$ particles. This may be explained by the fact that the drop in particle temperature within the nozzle and the nearfield plume regions scales roughly with the inverse of the particle diameter, following a balance between particle heat capacity and the heat transfer rate. As all particles enter the nozzle at similar temperatures, we can generally expect an increase in temperature with particle size throughout the plume.

Radiative heat transfer is shown in the figure to result in a continuous downstream reduction in temperature for 4 $\mu \mathrm{m}$ particles, up to a narrow region about $50 \mathrm{~m}$ away from the nozzle where these particles reach a temperature of $1930 \mathrm{~K}$. In our model for nonequilibrium phase change, this is specified as the nucleation temperature at which a crystallization front forms uniformly over the surface of an initially liquid particle. Once formed, this front progresses toward the particle center at a temperature dependent rate, so long as the particle temperature remains below the equilibrium melting temperature of $2327 \mathrm{~K}$. As the heat of formation for liquid $\mathrm{Al}_{2} \mathrm{O}_{3}$ is released during crystallization, particles will experience a rapid temperature increase at the initiation of the phase change process. The rate of this increase will be reduced further downstream as the particle temperature rises, so the crystallization front progresses more slowly, and as the front area decreases, so a smaller volumetric phase change rate will exist for a given velocity of progression toward the particle center. Ultimately, as the particle becomes completely solidified, we expect the particle temperature to again begin to decrease with downstream distance due to radiative heat loss. For $4 \mu \mathrm{m}$ particles, the temperature reduction following crystallization should occur beyond the downstream limit of the simulation domain, so this trend is not shown in Fig. 2. Note that the phase change process for $0.4 \mu \mathrm{m}$ particles is completed primarily within the nozzle, so no similar temperature jump is shown in the upper half of the figure.

In Fig. 3, particle temperature profiles are shown along the radial plane $100 \mathrm{~m}$ downstream of the nozzle exit. Average temperatures for particles of four different sizes are plotted as a function of distance from the axis, and for comparison, corresponding temperature profiles are shown for a simulation in which the radiation model is disabled. First consider the temperature profiles for the latter case: For all particle sizes shown, temperatures are found to increase with distance from the axis due to the radial variation in gas density within the plume nearfield, as discussed above. The temperature is also found to generally increase with particle size, so that the lowest 
temperatures are observed for the smallest particles shown, while the highest temperatures occur for the largest particles. An important exception to this trend is observed for $0.6 \mu \mathrm{m}$ diameter particles, which experience temperatures significantly higher than those of the much larger $4 \mu \mathrm{m}$ particles. This can be explained by characteristics of the crystallization process. While $4 \mu \mathrm{m}$ particles never undergo sufficient convective cooling to reach the nucleation temperature of $1930 \mathrm{~K}$ at which crystallization may begin, the $0.4 \mu \mathrm{m}$ and $0.6 \mu \mathrm{m}$ particles pass through the nozzle exit plane below the melting temperature and in a state of partial solidification. The entire phase transition process for $0.4 \mu \mathrm{m}$ particles takes place within and just beyond the nozzle, where convective heat loss dominates the particle energy balance and allows for temperatures several hundred degrees below the melting temperature of $2327 \mathrm{~K}$. In contrast, the phase change process for $0.6 \mu \mathrm{m}$ particles occurs at a slower rate, and continues well beyond the nearfield plume region where the associated heat release may be balanced by convective heat transfer to the gas. As a result, the temperature of these particles approaches a farfield limit which is significantly greater than that of both larger and smaller particles.

In comparing temperature profiles between the cases with and without activation of the radiation model, we find that radiative heat loss accounts for a roughly $120 \mathrm{~K}$ decrease in temperatures for $0.4 \mu \mathrm{m}$ particles and a $200 \mathrm{~K}$ decrease for $0.6 \mu \mathrm{m}$ particles. Among $4 \mu \mathrm{m}$ particles however, radiative heat transfer is found to increase temperatures $100 \mathrm{~m}$ downstream of the nozzle by about $240 \mathrm{~K}$. As above, this trend may be explained by the phase change process: Convective cooling within the nozzle and plume nearfield regions is not sufficient to reduce the temperature of $4 \mu \mathrm{m}$ particles to below the nucleation temperature, but the addition of radiative cooling results in farfield particle temperatures at which crystallization fronts may form. Because the rate of heat addition for a particle undergoing crystallization is far greater than the rate of radiative heat loss, these particles will experience a rapid temperature increase as shown in Fig. 2.

For $6 \mu \mathrm{m}$ particles, a comparison of temperature profiles shows an even more complicated trend. Radiation is found to uniformly reduce particle temperatures, but the magnitude of this reduction varies greatly with distance from the axis. This trend may be explained as follows: Radial variation in nearfield convective heat transfer results in an increase in temperature for these particles with distance from the axis, as described above. This means that particles further from the axis must experience radiative heat loss over a longer time period before their temperatures have been sufficiently reduced for crystallization to begin. The temperature rise associated with crystallization therefore occurs further downstream for particles at a greater distance from the axis. At the radial plane $100 \mathrm{~m}$ downstream of the axis, $6 \mu \mathrm{m}$ particles close to the centerline have begun the phase change process, while the fraction of particles on which crystallization fronts have formed is generally found to decrease with radial distance. This reduction in liquid mass fraction with radial distance corresponds to the temperature drop observed in Fig. 3 for $6 \mu \mathrm{m}$ particles far from the axis.

Figure 4 shows the centerline variation in the magnitude of mean convective and radiative heat transfer rates, as calculated per particle and averaged over all particle sizes. Both rates are negative through the entire simulation domain, so that particles throughout the plume are losing energy to their surroundings through both convective and radiative heat transfer. The radiative heat transfer rate is shown to be relatively constant with downstream distance, due to an overall gradual variation in particle temperatures and the fact that radiative absorption is found to have a comparatively small effect. Most of the variation in radiative transfer observed on the plot is the result of statistical scatter, due to the small number of representative particles which pass through cells bordering the axis. However, a significant increase in the radiative transfer rate is found about $50 \mathrm{~m}$ downstream of the nozzle exit. This may be attributed to a temperature jump associated with the onset of phase change in $4 \mu \mathrm{m}$ particles, which account for about $60 \%$ of the total $\mathrm{Al}_{2} \mathrm{O}_{3}$ mass within the plume.

In contrast to the relatively uniform rate of radiative heat transfer, the mean convective heat transfer rate is shown to decrease rapidly with downstream distance. The spatial variation in convective heat transfer is found to occur mainly as a result of a downstream decrease in gas density. As the gas approaches a free molecular state far from the nozzle, the gas density will decrease along the centerline as the inverse square of the distance from some point near the nozzle exit. The farfield convective heat transfer rate will therefore have the same inverse square of distance variation, as is shown in Fig. 4. A convenient definition of the plume nearfield region in a freely expanding SRM plume flow is the range beyond the nozzle exit where the particle energy balance is dominated by convective heat transfer to the gas. By this definition, the nearfield region extends along the centerline about $1 \mathrm{~m}$ (or 13 nozzle exit diameters) downstream of the nozzle exit, beyond which radiative emission becomes the dominant mechanism for energy transfer between a particle and its surroundings.

In Fig. 5, contours are shown for the net direction-averaged radiative energy flux. The energy flux is greatest at the nozzle exit, due to the corresponding maximum in particle mass density and a reduction in intensity of searchlight emission with distance from the nozzle. Particles are modeled through Eq. (1) as volumetric emitters, ${ }^{1}$ so 
the magnitude of radiative energy flux should scale roughly with the local particle mass density. As this density decreases with downstream distance due to the divergence of particle trajectories, a continuous reduction in radiative energy flux will occur in the axial direction.

A reduction in energy flux is also observed in the radial direction, particularly outside the region where particles are found. This follows primarily from the fact that in an axisymmetric simulation the ratio of cell volume to the projected area on the grid will scale with distance from the axis. The average number of energy bundles which pass through a cell is proportional to the projected area of that cell, and the contribution of each bundle to the energy flux is proportional to the inverse of the cell volume. We therefore expect the net energy flux through cells outside the particle domain to scale approximately with the inverse of the distance from the axis, as is shown in the figure.

Figure 6 shows a comparison of net radiative energy flux contours between simulations with and without activation of the model for nozzle searchlight emission. Due to the point-source nature of this effect, searchlight emission is found to have relatively little influence on energy flux magnitudes far downstream of the nozzle. For clarity we therefore restrict the figure to an axial range of $10 \mathrm{~m}$ from the nozzle exit. The presence of searchlight emission results in a $100 \%$ increase in radiative energy flux at the point along the centerline $0.5 \mathrm{~m}$ from the nozzle exit. At points 1, 2, 5 and $10 \mathrm{~m}$ downstream, we find corresponding increases of about $45 \%, 13 \%, 11 \%$ and $9 \%$, respectively. An equivalent contour plot to evaluate the impact of anisotropic scattering is included as Fig. 7. The top half of this figure shows contours of net direction-averaged energy flux for the base simulation, as described above, where we apply the Henyey Greenstein scattering phase function. The lower half of the figure is taken from a simulation where isotropic scattering is used, so that each scattered energy bundle is randomly assigned a new direction of propagation with no dependence on the initial direction. We find little if any measurable effect of the scattering model on energy flux contours, as the small differences observed between the upper and lower halves of Fig. 7 are primarily a result of statistical fluxuations due to the probabilistic nature of the radiation model.

Spectral radiance is calculated at a simulated radiometer centered at a point $20 \mathrm{~cm}$ upstream and $4 \mathrm{~cm}$ radially outward from the intersection of the central axis with the nozzle exit plane. The sensor has a circular face of area 50 $\mathrm{cm}^{2}$ and a surface normal vector inclined $4^{\circ}$ from the axis. The angular resolution is $4^{\circ}$, so that the boundary of the conical viewing area intersects the grid plane along a line which is parallel to the axis. Values of spectral radiance are plotted in Fig. 8, where the solid line denotes the base simulation and dashed lines correspond to simulations for which either searchlight emission or the anisotropic scattering model is disabled. All three lines generally follow the gray body-like trend described by Reed and Calia. ${ }^{1}$

As expected, we find that searchlight emission produces an increase in radiance values upstream of the nozzle. However, the magnitude of this increase is very small compared to the increase in radiative energy flux near the nozzle shown in Fig. 6. As discussed by Reed et al., ${ }^{16}$ the relatively small dependence of measured radiance on searchlight emission may be attributed to a strong preference for forward scattering among $\mathrm{Al}_{2} \mathrm{O}_{3}$ particles. Any nozzle emission absorbed by the sensor must be scattered off one or more particles at a large angle, for which the scattering efficiency is very low. Searchlight emission will therefore have a far smaller effect on radiance upstream of the nozzle than on radiance measured at a point downstream of the exit plane. If the angular dependence in the scattering model is disabled, then we expect to find a significant increase in radiance upstream of the nozzle due to searchlight emission. This trend is shown in Fig. 8, where a jump in spectral radiance of up to $20 \%$ is found when scattering is assumed to be isotropic.

\section{Conclusions}

A radiation model was presented for the $\mathrm{Al}_{2} \mathrm{O}_{3}$ particle phase in SRM plume flows at high altitude. The model includes capabilities for strong two-way coupling between radiation and flowfield calculations, and accounts for the influence of emission and absorption on particle temperatures. No limitations are imposed on optical thickness of the flowfield, and band-averaged particle radiative properties are allowed to vary as an arbitrary function of both temperature and wavelength. Procedures were described for the calculation of spectral radiance and consideration of effects associated with nozzle searchlight emission. Following application of the model in the axisymmetric simulation of a subscale plume flow, several radiation and flowfield characteristics were evaluated. A complex relationship was shown between particle size and temperature in farfield plume regions, due in part to the interaction between radiative heat loss and crystallization of liquid $\mathrm{Al}_{2} \mathrm{O}_{3}$. Searchlight emission was found to significantly affect the radiative energy flux through a large region beyond the nozzle exit, while consideration of the angular dependence in particle scattering results in an intensity reduction upstream of the nozzle.

Due to a lack of available experimental data for comparison, the overall accuracy of our radiation model in the simulation of a high altitude SRM plume flow could not be quantitatively evaluated. However, we expect that the single greatest error source is the selection of appropriate values for the particle absorption index, particularly at low 
temperatures where much of the particle material has solidified. As discussed by Reed and Calia, ${ }^{1}$ the absorption index of solid $\mathrm{Al}_{2} \mathrm{O}_{3}$ particles is primarily an extrinsic property, and is a strong function of the concentration of impurities within the lattice structure. As impurity concentrations will vary greatly between different SRM exhaust flows, and between different regions in the same flow, the identification of reasonably accurate values for the absorption index of solid $\mathrm{Al}_{2} \mathrm{O}_{3}$ becomes extremely difficult. Based on experimental measurements ${ }^{18}$ we can expect these values to be accurate to about one order of magnitude, so that radiative heat transfer rates and energy flux values far from the nozzle may vary on this same scale.

Another potential source of significant error is the difficulty in accurately determining particle properties at the nozzle exit. As a result of complicated physical processes which occur within the combustion chamber and nozzle, such as particle agglomeration, breakup, combustion, and turbulent dispersion, the accurate numerical calculation of particle properties at the exit plane may be very difficult. Other difficulties are associated with the experimental measurement of particle phase characteristics along the nozzle exit, and no sufficiently detailed experimental data could be found in the open literature. We must therefore rely on simplified nozzle flow simulations which neglect many of the physical phenomena expected in such flows, so that a loss of accuracy will result in the calculation of particle properties within the plume. Other possible sources of significant error in the radiation model include the lack of consideration for IR absorption and emission involving exhaust gas species, approximations used to compute particle emissivity and scattering coefficients, and an unphysical reduction in farfield energy fluxes and radiative heat transfer due to the truncation of the simulation domain.

While the radiation model presented here was developed for application to high altitude SRM plume flows, the above procedures may be applied to any simulation involving a Lagrangian representation of micron-scale $\mathrm{Al}_{2} \mathrm{O}_{3}$ particles. This includes the simulation of internal and external SRM exhaust flows at lower altitudes, where the gas phase may be accurately modeled using continuum CFD methods. To the authors' knowledge, this is the first implementation of a Monte Carlo ray trace model to allow for strong coupling between radiation and flowfield characteristics in a high speed emitting, absorbing and scattering multiphase medium of arbitrary optical thickness. With sufficient modifications, we expect that the general procedure described here may be applied to a variety of gas-particle flows.

\section{Acknowledgments}

The authors gratefully acknowledge the Air Force Research Laboratory at Edwards Air Force Base for financial support of this work, with Dean Wadsworth and Tom Smith as technical monitors.

\section{References}

${ }^{1}$ Reed, R. A., and Calia, V. S., “Review of Aluminum Oxide Rocket Exhaust Particles,” AIAA Paper 93-2819, 1993.

${ }^{2}$ Geisler, R. L., “A Global View of the Use of Aluminum Fuel in Solid Rocket Motors,” AIAA Paper 2002-3748, 2002.

${ }^{3}$ Bird, G. A., Molecular Gas Dynamics and the Direct Simulation of Gas Flows, Clarendon Press, Oxford, 1994.

${ }^{4}$ Gallis, M. A., Torczynski, J. R., and Rader, D. J., “An approach for Simulating the Transport of Spherical Particles in a Rarefied Gas Flow via the Direct Simulation Monte Carlo Method,” Physics of Fluids, Vol. 13, No. 11, 2001, pp. 3482-3492.

${ }^{5}$ Burt, J. M., and Boyd, I. D., "Development of a Two-Way Coupled Model for Two Phase Rarefied Flows," AIAA Paper 2004-1351, 2004.

${ }^{6}$ Burt, J. M., and Boyd, I. D., "Particle Rotation Effects in Rarefied Two Phase Plume Flows,” 24th International Symposium on Rarefied Gas Dynamics, Monopoli, Italy, 2004.

${ }^{7}$ Dietrich, S., and Boyd, I. D., "Scalar and Parallel Optimized Implementation of the Direct Simulation Monte Carlo Method,” Journal of Computational Physics, Vol. 126, 1996, pp. 328-342.

${ }^{8}$ Burt, J. M., and Boyd, I. D., "Monte Carlo Simulation of a Rarefied Multiphase Plume Flow,” AIAA Paper 2005-0964, 2005.

${ }^{9}$ Farmer, J. T., and Howell, J. R., "Monte Carlo Prediction of Radiative Heat Transfer in Inhomogeneous, Anisotropic, Nongray Media,” Journal of Thermophysics and Heat Transfer, Vol. 8, No. 1, 1994, pp. 133-139.

${ }^{10}$ Mahan, J. R., Radiation Heat Transfer: a Statistical Approach, John Wiley and Sons, New York, 2002.

${ }^{11}$ Plass, G. N., "Temperature Dependence of the Mie Scattering and Absorption Cross Sections for Aluminum Oxide," Applied Optics, Vol. 4, No. 12, 1965, pp. 1616-1619.

${ }^{12}$ Siegel, R., and Howell, J. R., Thermal Radiation Heat Transfer, Hemisphere Publishing, Washington, 1981.

${ }^{13}$ Laredo, D., and Netzer, D. W., “The Dominant Effect of Alumina on Nearfield Plume Radiation,” Journal of Quantitative Spectroscopy and Radiative Transfer, Vol. 50, No. 5, 1993, pp. 511-530.

${ }^{14}$ Everson, J., and Nelson, H. F., "Development and Application of a Reverse Monte Carlo Radiative Transfer Code for Rocket Plume Base Heating,” AIAA Paper 93-0138, 1993.

${ }^{15}$ Sun, Q., and Boyd, I. D., “Evaluation of Macroscopic Properties in the Direct Simulation Monte Carlo Method,” Journal of Thermophysics and Heat Transfer, submitted for publication, 2004. 
${ }^{16}$ Reed, R. A., Beale, K. S., Neese, D. W., Sherrell, F. G., Roberds, D. W., and Oliver, S. M., "The Effect of Seachlight Emission on Radiation from Solid Rocket Plumes,” AIAA Paper 92-2918, 1992.

${ }^{17}$ Anfimov, N. A., Karabadjak, G. F., Khmelinin, B. A., Plastinin, Y. A., and Rodinov, A. V., "Analysis of Mechanisms and Nature of Radiation from Aluminum Oxide in Different Phase States in Solid Rocket Exhaust Plumes,” AIAA Paper 93-2818, 1993.

${ }^{18}$ Konopka, W. L., Reed, R. A., and Calia, V. S., "Measurements of Infrared Optical Properties of Al2O3 Rocket Particles,” AIAA Paper 83-1568, 1983.

${ }^{19}$ Duval, R., Soufiani, A., and Taine, J., "Coupled Radiation and Turbulent Multiphase Flow in an Aluminised Solid Propellant Rocket Engine,” Journal of Quantitative Spectroscopy and Radiative Transfer, Vol. 84, No. 4, 2004 , pp. 513-526.

Table 1. Particle properties at the nozzle exit.

\begin{tabular}{|l|l|l|l|l|}
\hline $\begin{array}{l}\text { Diameter, } \\
\mu \mathrm{m}\end{array}$ & $\begin{array}{l}\text { Mass flux, } \\
\mathrm{kg} / \mathrm{m}^{2} \mathrm{~s}\end{array}$ & $\begin{array}{l}\text { Temperature, } \\
\mathrm{K}\end{array}$ & $\begin{array}{l}\text { Speed, } \\
\mathrm{m} / \mathrm{s}\end{array}$ & $\begin{array}{l}\text { Liquid mass } \\
\text { fraction }\end{array}$ \\
\hline 0.3 & 0.0443 & 1562 & 2992 & 0.579 \\
\hline 0.4 & 0.0367 & 1634 & 3051 & 0.661 \\
\hline 0.6 & 0.133 & 1834 & 3023 & 0.89 \\
\hline 1 & 0.592 & 2293 & 2973 & 1 \\
\hline 2 & 2.29 & 1920 & 2855 & 0.989 \\
\hline 4 & 7.53 & 2178 & 2674 & 1 \\
\hline 6 & 1.84 & 2407 & 2472 & 1 \\
\hline
\end{tabular}

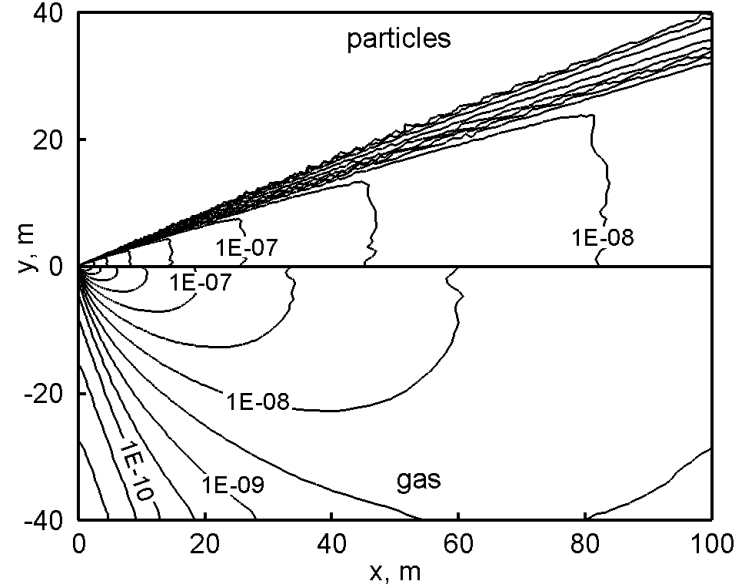

Figure 1. Contours of mass density for particles and gas. Values are given in $\mathrm{kg} / \mathrm{m}^{3}$.

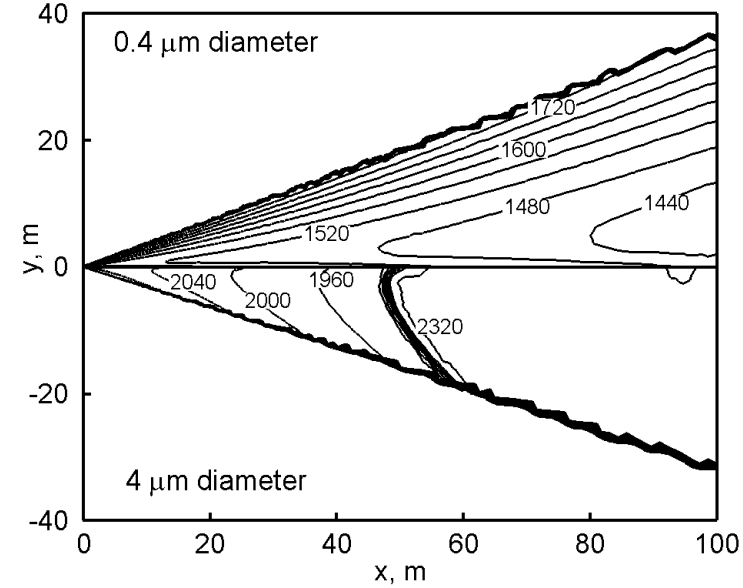

Figure 2. Mean temperature contours for $0.4 \mu \mathrm{m}$ and $4 \mu \mathrm{m}$ diameter particles. Values are in $\mathrm{K}$. 


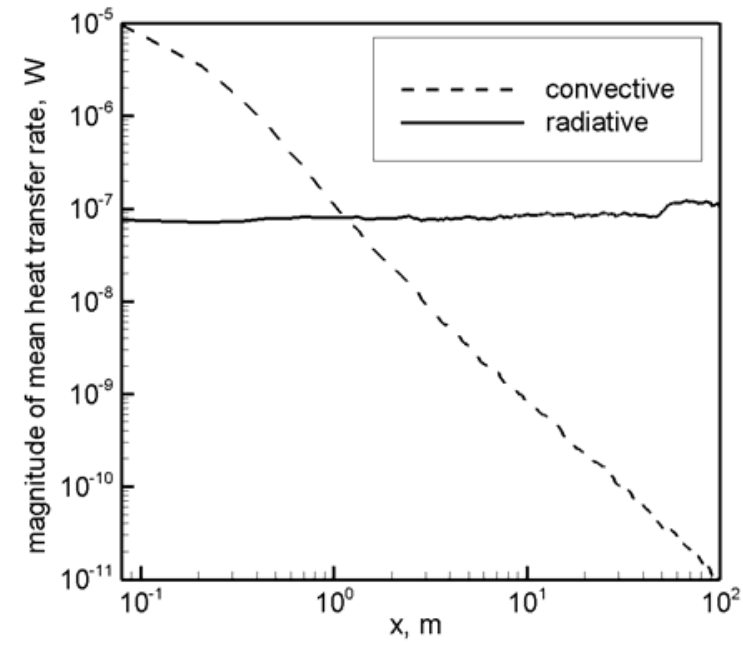

Figure 3. Profiles of particle temperature $100 \mathrm{~m}$ downstream of the nozzle exit.

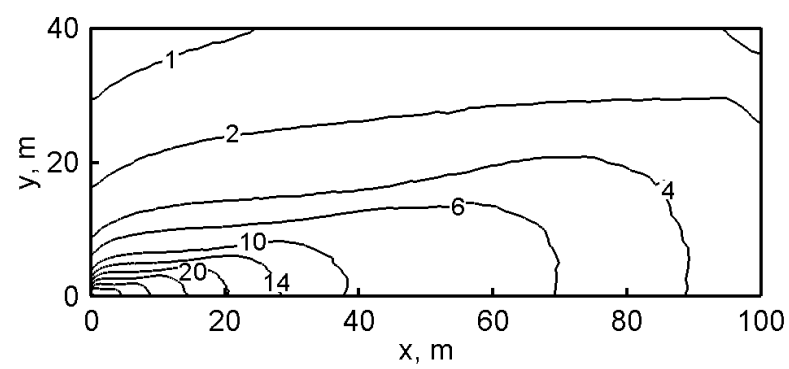

Figure 5. Contours of the net direction-averaged radiative energy flux. Values are in $\mathrm{W} / \mathrm{m}^{2}$.

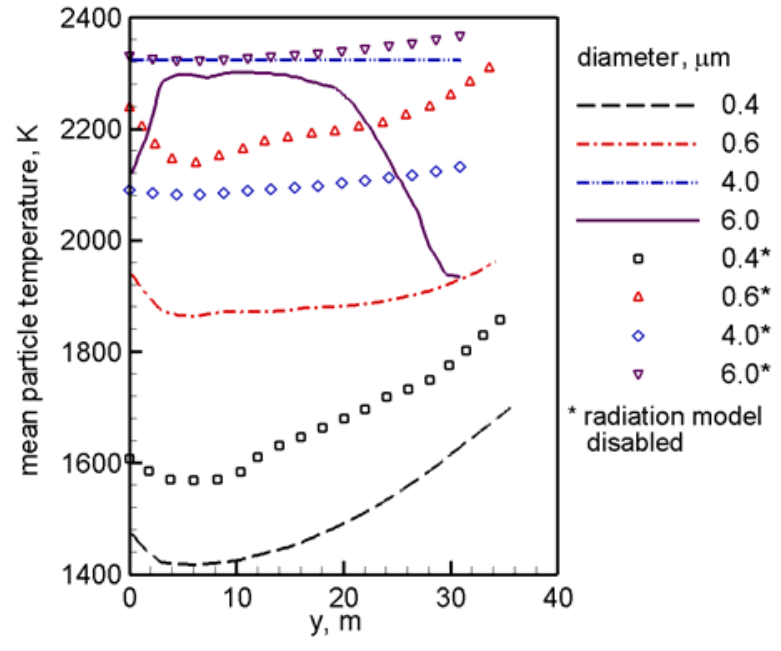

Figure 4. Mean convective and radiative heat transfer rates along the centerline.

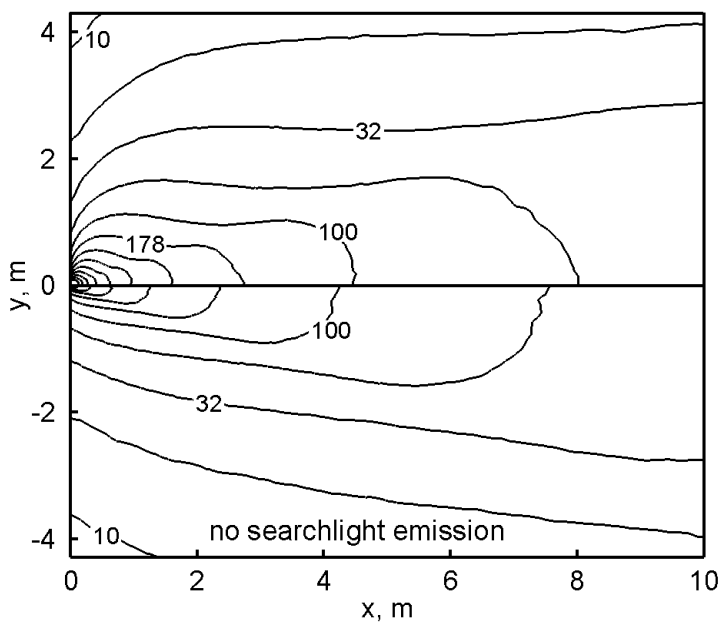

Figure 6. Comparison of the net radiative energy flux with and without searchlight emission. Values are in $\mathrm{W} / \mathbf{m}^{2}$. 


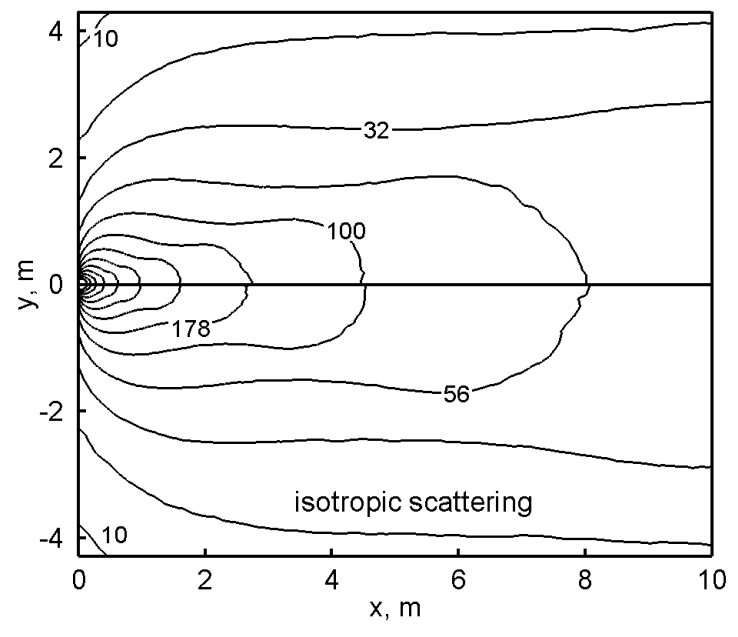

Figure 7. Energy flux contours for simulations employing anisotropic (top) and isotropic scattering models. Values are in $\mathrm{W} / \mathrm{m}^{2}$.

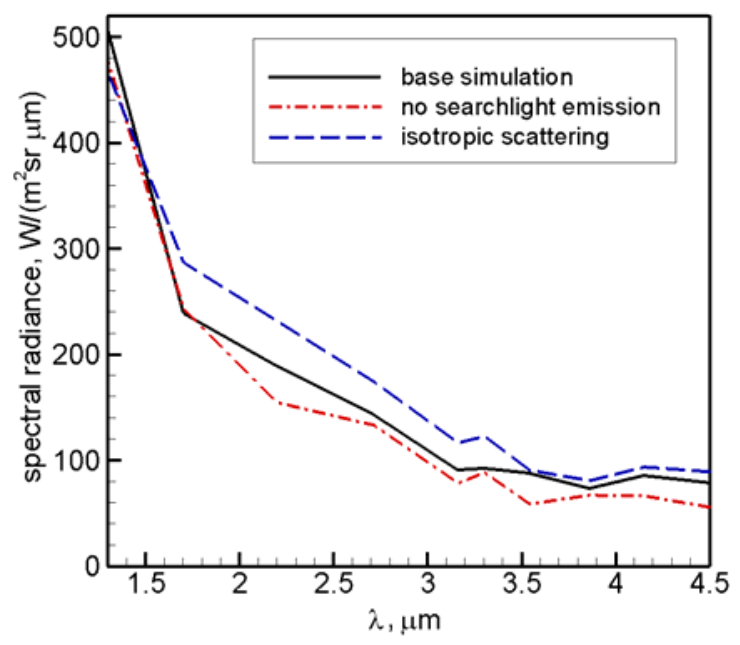

Figure 8. Spectral radiance at a sensor $20 \mathrm{~cm}$ upstream of the nozzle exit. 